ORIGINAL ARTICLE

\title{
Is antenatal syphilis screening still cost effective in sub-Saharan Africa
}

\author{
F Terris-Prestholt, D Watson-Jones, K Mugeye, L Kumaranayake, L Ndeki, H Weiss, \\ J Changalucha, J Todd, F Lisekie, B Gumodoka, D Mabey, R Hayes
}

See end of article for authors' affiliations

.....................

Correspondence to: Fern Terris-Prestholt, HIVTools Research Group, Health Policy Unit, London School of Hygiene and Tropical Medicine, Keppel Street, London WC1E 7HT, UK; Fern.Terris-Presthol+@ Ishtm.ac.uk

Accepted for publication 29 April 2003

\begin{abstract}
Objectives: To estimate the cost effectiveness of on-site antenatal syphilis screening and treatment in Mwanza, Tanzania. To compare this intervention with other antenatal and child health interventions, specifically the prevention of mother to child transmission of HIV (PMTCT).

Methods: The economic costs of adding the intervention to routine antenatal care were assessed. Cost effectiveness (CE) ratios of the intervention were obtained for low birth weight (LBW) live births and stillbirths averted and cost per DALY saved. Cost per DALY saved was also estimated for previous CE studies of syphilis screening. The CE of the intervention at different syphilis prevalence rates was modelled. Results: The economic cost of the intervention is $\$ 1.44$ per woman screened, $\$ 20$ per woman treated, and $\$ 187$ per adverse birth outcome averted. The cost per DALY saved is $\$ 110$ with LBW as the only adverse outcome. When including stillbirth, this estimate improves 10 -fold to $\$ 10.56$ per DALY saved. The cost per DALY saved from all syphilis screening studies ranged from $\$ 3.97$ to $\$ 18.73$.

Conclusions: Syphilis screening is shown to be at least as cost effective as PMTCT and more cost effective than many widely implemented interventions. There is urgent need for scaling up syphilis screening and treatment in high prevalence areas. The CE of screening interventions is highly dependent on disease prevalence. In combination, PMTCT and syphilis screening and treatment interventions may achieve economies of scope and thus improved efficiency.
\end{abstract}

S caling up of interventions to prevent mother to child transmission of HIV (PMTCT) is now dominating the antenatal policy agenda in settings with high HIV prevalence. ${ }^{12}$ However, other simple and cheap antenatal interventions are still not universally implemented. The 1993 World Development Report (WDR) cites antenatal syphilis screening as one of the most cost effective ways to improve children's health. ${ }^{3}$ Despite this, only an estimated 38\% of women attending for antenatal care in Africa receive syphilis screening. ${ }^{4}$

A high prevalence of syphilis continues to be found throughout sub-Saharan Africa, ranging between $2.5 \%$ in Burkina Faso, 8.4\% in South Africa, and $17.4 \%$ in Cameroon in 1999. ${ }^{5}$ Maternal syphilis is associated with numerous adverse pregnancy outcomes such as spontaneous abortion, stillbirth, low birth weight (LBW), premature delivery, and congenital syphilis. ${ }^{6}$ Maternal syphilis is estimated to contribute to $29 \%$ of perinatal deaths, $11 \%$ of neonatal deaths, and $26 \%$ of stillbirths around the world. ${ }^{7}$ Screening and treating mothers for syphilis during pregnancy can prevent adverse pregnancy outcomes associated with maternal infection. $^{8}$

Maternal syphilis screening and treatment are believed to be cost effective. ${ }^{9-12}$ Published cost effectiveness (CE) studies focusing on developing countries have relied on assumptions or historical data to estimate treatment effectiveness. None has calculated the CE of the intervention using a generic outcome indicator, such as disability adjusted life years (DALYs), by which comparisons with other interventions can be made. Limited data on the costs of implementation have been presented and some studies have relied on modelled hypothetical costs rather than providing real values. ${ }^{9} 12$

We examined the CE of on-site antenatal syphilis screening and treatment in Mwanza city, Tanzania. Pregnant women in Mwanza have an estimated prevalence of active syphilis of
$8 \%$ and an HIV prevalence of $10-11 \% .^{813}$ This study addressed some of the limitations of cost and outcome measures used in earlier CE studies of antenatal syphilis screening. To provide a more reliable estimate of effectiveness, our analysis used the number of adverse outcomes averted by the intervention, based on observed rates of adverse birth outcomes in Mwanza Region in women who had untreated maternal syphilis and in women who were screened and treated for syphilis. The incremental costs of adding syphilis screening to routine antenatal care were estimated and the cost per DALY saved was calculated. The robustness of these estimates was explored with univariate sensitivity analysis around key assumptions used in the study. We recalculated the CE from previous studies of syphilis screening using our methodology and compared this with the existing evidence base of the CE of other antenatal and child health interventions. The impact of syphilis prevalence on CE was also modelled.

\section{METHODS}

\section{The intervention}

In 1997 the Tanzanian Ministry of Health and the African Medical and Research Foundation (AMREF) introduced onsite rapid syphilis screening and treatment with a single dose of benzathine penicillin into routine antenatal care at the main antenatal clinic (ANC) in Mwanza. The syphilis screening programme (known as the intervention) aimed to screen all new attenders for syphilis as part of routine antenatal services. Women were screened if they presented for their first antenatal visit in that pregnancy, or if they had previously attended an ANC that did not offer screening. Women gave a blood sample that was tested by the rapid plasma reagin (RPR) test. All RPR positive women were treated on the same day with a single dose of benzathine penicillin 2.4 MU by intramuscular injection. They were 
individually counselled on the results. RPR positive mothers were encouraged to send their partners for treatment.

From September 1997 to November 1999 an evaluation of the effectiveness of screening and treatment with single dose benzathine penicillin in averting adverse birth outcomes was conducted. The results of this study provided the cost and cost effectiveness measures for this analysis. ${ }^{8}$

\section{Costs}

Cost collection was based on the Costing Guidelines for HIV Prevention Strategies. ${ }^{14}$ Costs were collected retrospectively using an ingredients based costing methodology. The total costs of implementing the intervention were collected, including relevant activities undertaken by the municipal health services, the research study, and AMREF. Resources used from July 1998 through June 1999 were compiled to determine the annual costs of running the intervention. Both financial and economic costs were estimated from a provider's perspective. Financial costs represent actual project expenditure. Economic costs represent the estimated market value of all resources used in the intervention, including donated goods and services. As the intervention was added to existing ANC services, incremental costs were collected. The cost data were collected from administrative records in the health clinic, the research project accounts, and from the AMREF office in Mwanza, as well as through discussions with the project staff and observation at the ANC site. Costs for the start up and implementation of the intervention were included in the analysis. This also included treating partners who presented for treatment. Costs associated with pure research related activities were excluded. Although during the evaluation, quality control was carried out by the study team, the cost of this was not included in our analysis because, before the evaluation, the intervention had abandoned routine quality control because of operational difficulties.

The annual costs of capital goods were calculated using straight line depreciation for financial costs and annualisation with a $3 \%$ discount rate for economic costs. Capital goods were given an estimated life between 3 and 10 years. Equipment included an RPR shaker, a refrigerator, and a vacutainer centrifuge. Primary personnel involved were laboratory staff, a counsellor, a nurse, and a team leader.

The average annual exchange rate in the year of the expenditure was used to convert Tanzanian shillings into US\$, ranging from 619 to 749 Tanzanian shillings per US\$. All costs presented in this paper, including costs quoted from other studies, have been adjusted to 2001 US\$. Given the retrospective nature of the cost data collection, it was only possible to estimate the proportion of time spent by staff on the various intervention activities. The impact of this estimation was explored in the sensitivity analysis.

\section{Effectiveness}

Data to measure the effectiveness of the intervention for this CE study came from two studies in Mwanza Region. The methodology for these studies has been previously published. ${ }^{8}{ }^{15}$ In summary, to measure the impact of untreated maternal syphilis on birth outcome, a retrospective cohort of 382 women was recruited at three hospitals in Mwanza Region. ${ }^{15}$ At delivery, consenting women, who had not been screened for syphilis during pregnancy, were screened for syphilis by the RPR test. Maternal sera from enrolled women were also tested by the Treponema pallidum haemagglutination assay (TPHA). If the TPHA test was negative and the RPR test was positive, sera were also tested by the fluorescent treponemal antibody assay (FTA). Syphilis stages were defined as high titre active syphilis (HTAS) when the RPR was reactive at a dilution of $\geqslant 1: 8$, low titre active syphilis
(LTAS) when the RPR titre was reactive at a titre of $<1: 8$, and old or resolved/treated syphilis when the RPR was nonreactive but the TPHA was positive. ${ }^{815} 16$ This definition was adopted in order to facilitate comparison with other African studies and because clinically based definitions could not be confirmed with available diagnostic tests in Mwanza. Of the 140 RPR positive women, $21 \%$ had a titre $<1: 8,19 \%$ had a titre $\geqslant 1: 8$ and $<1: 32$, and $34 \%$ had titres $\geqslant 1: 32$. Twenty six per cent of these women had a biological false positive RPR reaction shown by negative TPHA and FTA tests. Data were collected on stillbirth and LBW (which included prematurity) and cord blood serology. The study showed that women with untreated HTAS had a risk of stillbirth 18 times higher than women without syphilis, and a fourfold risk of any adverse outcome. ${ }^{15}$ No association was found between other stages of syphilis and adverse birth outcomes.

To measure the effectiveness of syphilis screening and treatment with single dose benzathine penicillin, a prospective cohort of 556 RPR positive and 1132 RPR negative ANC attenders was recruited and followed to delivery. On average, women presented for their first ANC visit in their 25th week of pregnancy. New ANC attenders were screened routinely using the RPR test. Of these women, 7.7\% were RPR positive and were treated with a single dose of 2.4 million units of benzathine penicillin intramuscularly at the same visit. Twenty seven per cent had HTAS. Infants were examined at birth for stillbirth and LBW including prematurity. The study showed that women screened and treated at the ANC for HTAS or LTAS had no increased risk of adverse pregnancy outcomes compared with seronegative women. ${ }^{8}$ This provided strong evidence that the intervention was effective in averting adverse outcomes attributable to maternal syphilis, since the first study had shown that approximately $49 \%$ of untreated women with HTAS from Mwanza Region experienced an adverse birth outcome. ${ }^{15}$

The cohort study did not include an infant follow up study and would not have had untreated infants to follow up to measure infants developing clinical congenital syphilis. Although all infants in the study were examined at birth, it is rare for an infected infant to show evidence of congenital syphilis at birth. ${ }^{6}$ Most symptoms of congenital syphilis appear from the age of 3 weeks and older. Published literature suggests that $14 \%$ to $50 \%$ of infants born to infected mothers will develop congenital syphilis, ${ }^{79}$ but there is little evidence about the proportion of infants with positive serology and a normal birth weight who develop clinical congenital syphilis. As it was not clear how much of the burden of disease was already taken into account by DALYs saved by averting low birth weight, this study has assumed that no cases of congenital syphilis were averted. This assumption is explored in the sensitivity analysis.

\section{Cost effectiveness}

Based on analysis of data from the 26 month prospective cohort study, effectiveness data for the CE study were matched for the year of the cost study. During this period, 9713 women were screened of which 696 (7.2\%) were RPR positive, and 264 partners presented for treatment at the ANC (0.38 partners per woman). The number of adverse outcomes averted was estimated by comparing the results of the two studies described above and are presented in table $1 .{ }^{8} 15$ Of women with a positive RPR test at the ANC, $26.8 \%$ (187 women) were estimated to have HTAS. ${ }^{17}$ During the period of the costing, the intervention prevented 44 stillbirths and 31 LBW infants, averting 75 adverse birth outcomes in total.

DALYs were calculated following the methodology described by Fox-Rushby and Hanson. ${ }^{18}$ DALYs are defined as the sum of years of life lost (YLL) due to premature 


\begin{tabular}{|c|c|c|c|c|}
\hline \multicolumn{5}{|l|}{ A } \\
\hline Adverse birth outcomes & \multicolumn{2}{|l|}{ Stillbirth } & \multicolumn{2}{|c|}{ Low birth weight } \\
\hline $\begin{array}{l}\text { Numbers susceptible } \\
\% \text { adverse outcome } \\
\text { Attributable fraction }{ }^{* *} \\
\text { Adverse birth outcomes averted } \neq\end{array}$ & \multicolumn{2}{|c|}{$\begin{array}{l}187 \text { (HTAS cases) }^{*} \\
25 \% \\
94 \% \\
44\end{array}$} & \multicolumn{2}{|c|}{$\begin{array}{l}140 \text { (live births) } \\
33 \% \\
67 \% \\
31\end{array}$} \\
\hline \multicolumn{5}{|l|}{ B } \\
\hline \multirow[b]{2}{*}{ DALYs } & \multicolumn{4}{|c|}{ Life expectancy } \\
\hline & Tanzania & WDR† & Tanzania & WDRT \\
\hline \multicolumn{5}{|l|}{ Years of life lost (YLL) } \\
\hline $\begin{array}{l}\text { YLL DALYs per case } \\
\text { Number }(\%) \text { deaths }\end{array}$ & 27.25 & 32.40 & $\begin{array}{l}27.25 \\
4(13 \%)\end{array}$ & 32.40 \\
\hline Number (\%) deaths & $44(100 \%)$ & & & \\
\hline \multicolumn{5}{|l|}{ Years lived with disability (YLD) } \\
\hline YLD DALYs per case & 0 & 0 & 7.93 & 9.42 \\
\hline Numbers (\%) of cases disabled & $0(0 \%)$ & & $1.9(6 \%)$ & \\
\hline Total YLD DALYS§ & 0 & 0 & 15 & 18 \\
\hline DALYS saved & 1195 & 1420 & 127 & 151 \\
\hline \multicolumn{5}{|l|}{ c } \\
\hline & \multicolumn{4}{|c|}{ Stillbirth and LBW } \\
\hline & \multicolumn{2}{|l|}{ Tanzania } & \multicolumn{2}{|l|}{ WDR* $^{*}$} \\
\hline Total DALYs saved & \multicolumn{2}{|l|}{1321} & \multicolumn{2}{|l|}{1571} \\
\hline \multicolumn{5}{|c|}{$\begin{array}{l}\text { *Syphilis was defined as HTAS when the RPR was reactive at a dilution of } \geq 1: 8 \text {. } \\
{ }^{*} \text { The estimated portion of adverse events in women with HTAS that were attributable to syphilis. } \\
\text { tWorld Development Report } 19933^{3} \\
\text { tAdverse birth outcomes }=\text { numbers susceptible } \times \text { percentage adverse outcomes } \times \text { attributable fractions. } \\
\text { \$YLL }=\text { YLL DALYs per case } \times \text { number of deaths; YLD }=\text { YLD DALYs per case } \times \text { number of cases disabled }\end{array}$} \\
\hline
\end{tabular}

mortality and years lived with disability (YLD) adjusted for severity. ${ }^{19}$ DALYs were calculated using adverse outcomes averted from the above studies. ${ }^{8}{ }^{15}$

In the past, stillbirth has not been included in DALY calculations because life expectancy is considered to begin at live birth. ${ }^{20}$ However, because stillbirth is the most important adverse outcome of untreated maternal syphilis in Mwanza Region, ${ }^{15}$ we have included DALYs saved by averting stillbirths, where stillbirth was considered a full life lost. DALYs saved are presented including and excluding stillbirth.

Sequelae of adverse pregnancy outcomes were taken from published literature. $.^{15} 21-25$ Of LBW infants, approximately $13 \%$ would have died and 6\% would have experienced a lifelong disability with a disability weight of 0.291 (the greater the weight the more severe the disability; a weight of 0 is no disability). ${ }^{18}$ Tanzanian life expectancy at birth (45 years), and the life expectancy at birth used in the WDR (81.75 years) were used to facilitate comparison with other studies. $^{326}$

A univariate sensitivity analysis was performed varying the main assumptions and inputs in the analysis. Personnel time inputs were reduced by $10 \%$ and increased by $20 \%$. The study coordinator's input was varied between $2 \%$ and $5 \%$ of her time in the recurrent costs, and the discount rate was varied from $1 \%$ to $6 \%$ in both the cost and DALY calculation. As the distribution of syphilis titres differed between the retrospective and the prospective studies, we explored the impact of $53 \%$ and $20 \%$ of women who tested RPR positive had HTAS. The mortality rates of LBW infants are highly variable. We used rates ranging from $10 \%$ to $40 \%{ }^{21-25}$ Although we assumed no cases of congenital syphilis were averted, we explored the effect of averting congenital syphilis if $14 \%$ and $50 \%$ of infants born to untreated mothers developed congenital syphilis and none of the infants born to treated mothers did. LBW and congenital syphilis were assumed not to be mutually exclusive, therefore a LBW infant could also develop CS. DALYs saved were DALYS saved from LBW cases averted plus DALY saved from CS cases averted.

\section{RESULTS}

\section{Incremental costs}

Table 2 presents the total annual costs, outcome indicators and average costs. The incremental economic costs were $\$ 13956,1 \%$ higher than financial costs of $\$ 13852$. As these are so similar, only detailed economic costs are presented. Personnel and supplies accounted for $91 \%$ of total costs. More than $60 \%$ of capital costs were attributable to start up activities.

The average costs were calculated by dividing the annual incremental economic costs by the outcome indicators. The cost per woman screened was $\$ 1.44$, the cost per woman treated was $\$ 20.05$, and the cost per person (women and partners) treated was $\$ 14.53$. The cost per averted stillbirth was $\$ 318.37$. The cost per LBW averted was $\$ 451.18$. The cost per all adverse outcomes averted was $\$ 186.66$. The CE of the intervention in preventing LBW, as measured by the cost per DALY saved, was $\$ 110.03$ and \$92.56, using the Tanzanian life expectancy and the WDR life expectancy, respectively. When including stillbirths, the cost per DALY saved was $\$ 10.56$ or $\$ 8.88$, depending on the life expectancy.

\section{Sensitivity analysis}

Reducing the DALY discount rate to $1 \%$ improved the CE by $36 \%$, and increasing the discount rate to $6 \%$ worsened the CE by $79 \%$ (table 3 ). The percentage of RPR positive women with HTAS had a large impact on cost effectiveness. An increase in the percentage of HTAS in RPR positives to $53 \%$ would decrease the cost per DALY saved including stillbirth to $\$ 5.43$, a $49 \%$ improvement. The mortality rate from LBW was 


\begin{tabular}{|c|c|c|}
\hline \multirow[b]{2}{*}{ Cost category } & \multicolumn{2}{|c|}{ Annual incremental economic costs } \\
\hline & $\$^{*}$ & $\%$ \\
\hline \multicolumn{3}{|l|}{ Capital } \\
\hline Buildings & 0 & 0 \\
\hline Equipment & 310 & 2.2 \\
\hline Vehicles & 0 & 0 \\
\hline Training & 110 & 0.8 \\
\hline Start up & 670 & 4.8 \\
\hline Total capital costs & 1090 & 7.8 \\
\hline \multicolumn{3}{|l|}{ Recurrent } \\
\hline Personnel & 5181 & 37.1 \\
\hline Supplies & 7546 & 54.1 \\
\hline Transportation costs & 139 & 1.0 \\
\hline $\begin{array}{l}\text { Building operating and } \\
\text { maintenance }\end{array}$ & 0 & 0 \\
\hline \multirow{3}{*}{$\begin{array}{l}\text { Total recurrent costs } \\
\text { Total costs }\end{array}$} & 12866 & 92.2 \\
\hline & 13956 & 100 \\
\hline & $\begin{array}{l}\text { Outcome } \\
\text { indicators }\end{array}$ & Average costs $\$^{*}$ \\
\hline Women screened & 9713 & 1.44 \\
\hline RPR positive women treated & 696 & 20.05 \\
\hline $\begin{array}{l}\text { People treated (women and } \\
\text { partners) }\end{array}$ & 960 & 14.53 \\
\hline $\begin{array}{l}\text { Cases of HTAS treated in } \\
\text { woment }\end{array}$ & 187 & 74.82 \\
\hline LBW live births averted & 31 & 451.18 \\
\hline Stillbirths averted & 44 & 318.37 \\
\hline $\begin{array}{l}\text { All adverse outcomes (LBW and } \\
\text { stillbirth) averted }\end{array}$ & 75 & 186.66 \\
\hline DALYs saved (LBW only) - TZ $\ddagger$ & 127 & 110.03 \\
\hline $\begin{array}{l}\text { DALYs saved (LBW only) - } \\
\text { WDR§ }\end{array}$ & 151 & 92.56 \\
\hline $\begin{array}{l}\text { DALYs saved (all adverse } \\
\text { outcomes) - TZ } \ddagger\end{array}$ & 1321 & 10.56 \\
\hline $\begin{array}{l}\text { DALYY saved (all adverse } \\
\text { outcomes) - WDRß }\end{array}$ & 1571 & 8.88 \\
\hline \multicolumn{3}{|c|}{$\begin{array}{l}\text { *Reported in year } 2001 \text { US } \$ \text {. Exchange rate: } 1 \$=815 \text { Tanzanian } \\
\text { shillings. } \\
\text { †Syphilis was defined as HTAS when the RPR was reactive at a dilution of } \\
\geqslant 1: 8 \text {. } \\
\text { †TZ = Tanzanian life expectancy in } 2000 \text {. } \\
\text { \$WDR = life expectancy used in the World Development Report, } 1993 .\end{array}$} \\
\hline
\end{tabular}

important in the cost per DALY saved due to LBW births averted, worsening CE by $28 \%$, and improving it by $64 \%$ with a lowering of the mortality to $10 \%$ and an increase to $40 \%$,

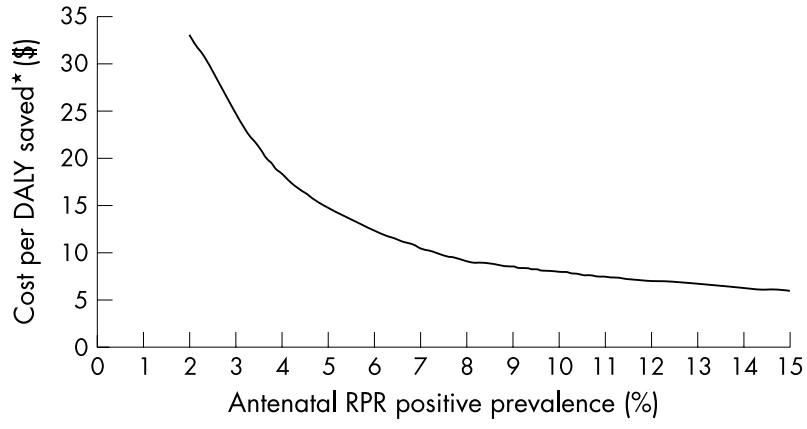

Figure 1 The relation between prevalence and cost effectiveness. *"Cost per DALY saved" includes stillbirth.

respectively. The impact on cost per DALY saved was far smaller when stillbirth was included, the divergence from the central estimate ranging from $2 \%$ to $-15 \%$. If the intervention averted $14 \%$ to $50 \%$ incidence of congenital syphilis, the CE would improve by $31 \%$ to $62 \%$, but only improved CE by $4 \%$ to $13 \%$ in the CE estimate including stillbirth. The CE estimates were not as sensitive to changes in the cost assumptions, ranging between $-6 \%$ and $16 \%$ divergence from the central estimate.

\section{The role of prevalence}

Cost effectiveness of the intervention is influenced by syphilis prevalence. We estimated the effect of different levels of syphilis prevalence (between $2 \%$ and $15 \%$ ) on the cost effectiveness of the intervention. The cost per DALY saved decreases substantially as prevalence increases. However, even at RPR prevalences as low as $2 \%$ antenatal syphilis screening and treatment remains cost effective (\$33 per DALY saved including stillbirth; fig 1).

\section{DISCUSSION}

The cost per DALY saved by the intervention in Mwanza ranged from $\$ 5.34$ to $\$ 196.63$. A detailed breakdown of the cost by input type was provided in order to consider the cost of replicating the intervention elsewhere. The effectiveness of the intervention was estimated in the same region over the same period using the Mwanza syphilis study results. This should provide a more reliable estimate of the intervention's

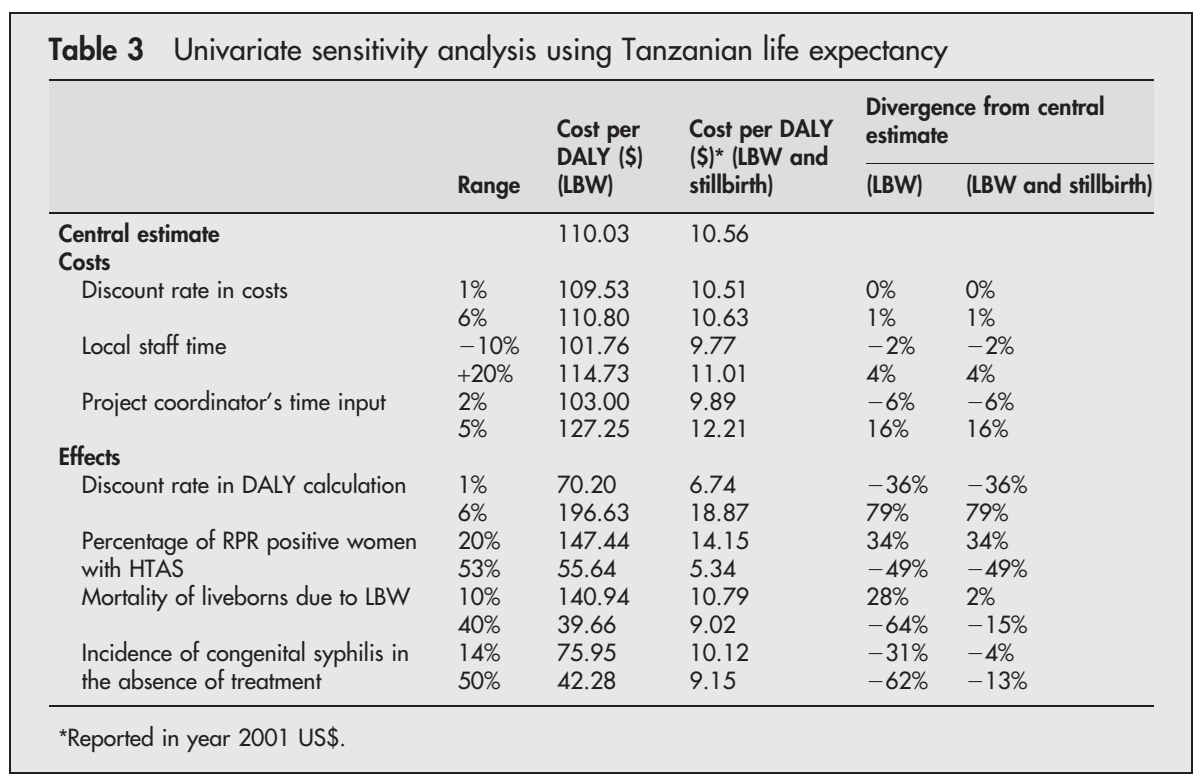


effectiveness in this population than using assumptions of effectiveness based on historical data.

\section{Comparison of cost effectiveness studies}

As none of the comparison studies included building costs, these studies were interpreted as providing costs for adding antenatal syphilis screening on to routine antenatal services. Hence these costs were interpreted as incremental costs. Jenniskens et al estimated the recurrent costs of on-site antenatal syphilis screening in Nairobi to be $\$ 34$ per case treated, ${ }^{10}$ including treating 0.57 partners per woman, which is more than double the cost incurred here. However, costs included a yearly refresher course as well as quality control, in contrast with this study where systematic quality assurance mechanisms were not in place. In the Jenniskens et al study, there was no breakdown of the costs, they did not include start up costs, and it is unclear whether capital costs were included. They assumed $40 \%$ of babies born to RPR positive women would contract congenital syphilis, while none of the babies born to treated women would. The cost per case of congenital syphilis averted was $\$ 62$. If we had calculated the numbers of congenital syphilis cases averted in the same way, we would have found a similar cost $(\$ 50$ per case averted).

Fonck et al examined the CE of this same syphilis screening programme after 6 years. ${ }^{11}$ More detailed costs were presented, but neither the costs of initial staff training nor clinic staff salaries were included. The source of the effectiveness assumptions was not clear. The sensitivity analysis from our study suggests that the variation in effectiveness is highly significant in determining CE, and so places even greater importance on ensuring appropriate measurement of outcome variables.

Hira et al estimated the incremental CE of a syphilis screening programme that included health education and attempted to screen women twice during pregnancy in Zambia." The CE estimate assumed $100 \%$ of RPR positive women were treated, although only $46 \%$ of their cohort actually received treatment. Using the actual numbers treated more than doubled their cost per adverse outcome averted ( $\$ 44$ per adverse outcome averted rather than \$19). In addition, it appears that they modelled the costs, rather than costing the actual project as implemented, and did not include any training or labour costs. Including the same portion of labour as used in Mwanza the cost per adverse outcome averted would rise to $\$ 70$. Key limitations of all the above studies are their assumptions on the number of adverse outcomes averted. Comparison of these three studies showed that as syphilis prevalence rises, the costs effectiveness of the intervention improved.

Our study is compared with the other three existing studies in table 4 . The left side of the table presents data from these studies, and uses them to calculate DALYs saved. Cost effectiveness for the three studies is modelled as if it were implemented in Mwanza, using effectiveness parameters from the current study on the right side. A number of critical assumptions are highlighted in table 4 . The stage of syphilis assumed to affect pregnancy outcome varied for the different studies. The study of untreated maternal syphilis in Mwanza found no association between LTAS or biological false positive reactions and adverse birth outcomes. ${ }^{15}$ We therefore only included women with HTAS in our calculations, $27 \%$ of those women treated at the ANC. Although the retrospective cohort had a higher percentage of RPR positive women with HTAS, this group is not representative of antenatal clinic attenders in terms of their treatment seeking behaviour. Hira and Jenniskens assumed all births of untreated RPR positive women would be affected, and Fonck assumed $69 \%$ would be affected. ${ }^{9-11}$ These studies assumed that $14 \%, 33 \%$ and $40 \%$ of children born live to an infected mother develop clinical congenital syphilis. ${ }^{9-11}$ We assumed no cases of congenital syphilis were averted.

The CE of the intervention in Tanzania falls between that of the Kenyan and Zambian studies when outcomes are measured by process indicators (number of women screened and numbers of people treated). The RPR prevalence in Mwanza also lies between the Kenyan and Zambian RPR prevalences. The intervention would need to improve its partner presentation rate from 0.38 partners per woman to 0.68 partners per woman to match the cost per person treated found in Zambia. There is a difference in cost per adverse outcome averted because of the other studies modelled adverse outcomes averted, while the Mwanza study measured the proportion of adverse outcomes in untreated mothers and assumed that these were all averted in treated mothers because they were not at increased risk compared to seronegative women. Using the effectiveness parameters from the Mwanza studies, these studies may have underestimated the cost per DALY saved by a factor of 2 to 3 , depending on the distribution of syphilis titres in their RPR positive population.

Despite the range of CE estimates (between $\$ 4$ and $\$ 33$ per DALY saved depending on RPR prevalences, titre distributions and outcome assumptions, and up to \$110 for DALYs saved by averting LBW alone), all fall below the suggested upper bound of CE interventions given by the WDR $(\$ 193)$. $^{3}$

\section{Comparison with other interventions}

The costing of nevirapine treatment for the PMTCT in Uganda with an ANC HIV seroprevalence of $15 \%$ estimates the incremental cost per DALY saved to be $\$ 11 .^{2}$ The syphilis screening intervention also compares well with other child health interventions. Cost effectiveness estimates for immunisations and oral rehydration therapy range from \$17-42 per DALY and \$7 and \$28 per DALY respectively. ${ }^{27}$

The CE calculations of this study used recent estimates of infections averted. Only adverse outcomes that were found to be associated with untreated maternal syphilis in Mwanza and averted by syphilis screening and treatment (stillbirth and LBW) were considered in this analysis. Neither congenital syphilis averted nor adult infections treated were taken into account. Syphilis screening and treatment also provides an opportunity to identify and counsel couples who may be at increased risk for HIV infection, thereby possibly preventing HIV infections. These make the current CE study more conservative.

There has been some debate about the construction of the DALY for antenatal interventions, and criticism that the WDR approach is too restrictive. ${ }^{20}$ The inclusion of stillbirth in constructing DALYs differentiates this study from other ANC syphilis screening and treatment studies as well as other maternal-child health interventions.

The CE of maternal syphilis screening is well within the range considered acceptable for other antenatal and child health interventions. The CE of screening and treatment is highly dependent on the prevalence of syphilis, with the cost per DALY saved decreasing with higher prevalences. If having to make choices between implementing PMTCT and syphilis screening programmes, it is critical to take the prevalence of HIV and syphilis into account. "Competition for resources and attention between high effect interventions for child survival and MTCT will not save children's lives. Building on the potential synergies of shared service delivery mechanisms for child survival and MTCT will save lives." ${ }^{28}$ Within the 
Table 4 Comparison of cost effectiveness studies

\begin{tabular}{|c|c|c|c|c|c|c|c|}
\hline & \multicolumn{3}{|l|}{ In articles } & \multirow[b]{2}{*}{ This study } & \multicolumn{3}{|c|}{ Using this study's effectiveness } \\
\hline & Hira et af & Jenniskens et al ${ }^{0}$ & Fonck et $a l^{11}$ & & Hira ef af & Jenniskens et al ${ }^{10}$ & Fonck et $a f^{11}$ \\
\hline Numbers screened & 806 & 13131 & 27377 & 9713 & 806 & 13131 & 27377 \\
\hline Number RPR positive (prevalences) & $74(9 \%)$ & $860(7 \%)$ & $928(3 \%)$ & $696(7 \%)$ & $74(9 \%)$ & $860(6 \%)$ & $928(3 \%)$ \\
\hline Number of RPR positive women treated & 34 & 751 & 845 & 696 & 34 & 751 & 845 \\
\hline Number of partners treated per woman & 0.85 & 0.57 & 0.53 & 0.38 & 0.85 & 0.57 & 0.53 \\
\hline $\begin{array}{l}\text { Percentage with syphilis stage assumed to } \\
\text { affect pregnancy outcome }\end{array}$ & 100 & 100 & 69 & 27 & 27 & 27 & 27 \\
\hline Number of pregnancies affected by treatment & 34 & 751 & 583 & 187 & 9 & 202 & 249 \\
\hline Number of live births & & & & 140 & 7 & 151 & 187 \\
\hline Effectiveness of treatment & $100 \%$ & $100 \%$ & $100 \%$ & $100 \%$ & $100 \%$ & $100 \%$ & $100 \%$ \\
\hline \multicolumn{8}{|l|}{ Expected percentage of adverse outcomes } \\
\hline Abortions & 17 & 0 & & & & & \\
\hline Stillbirths & 19 & & & 23 & 23 & 23 & 23 \\
\hline Stillbirth and prematurity & & $15^{*}$ & $33^{*}$ & & & & \\
\hline LBW & & & & 22 & 22 & 22 & 22 \\
\hline $\begin{array}{l}\text { Congenital syphilis } \\
\text { Number of outcomes averted }\end{array}$ & 14 & 40 & 33 & 0 & 0 & 0 & 0 \\
\hline Abortions & 6 & 0 & 0 & 0 & 0 & 0 & 0 \\
\hline Stillbirths & 6 & $56^{*}$ & $96^{*}$ & 44 & 2 & 47 & 58 \\
\hline LBW & 0 & $56^{*}$ & $96^{*}$ & 31 & 2 & 45 & 55 \\
\hline Congenital syphilis & 5 & 300 & 192 & 0 & 0 & 0 & 0 \\
\hline All adverse outcomes & 17 & 413 & 385 & 75 & 4 & 92 & 114 \\
\hline $\begin{array}{l}\text { Total DALYs saved } \\
\text { Costst }\end{array}$ & 190 & 2615 & 3560 & 1321 & 67 & 1472 & 1819 \\
\hline \multicolumn{8}{|l|}{ Costst } \\
\hline $\begin{array}{l}\text { lotal costs } \\
\text { Cost per woman screened }\end{array}$ & $\begin{array}{l}152 \\
093\end{array}$ & $\begin{array}{l}25 / 32 \\
1.96\end{array}$ & $\begin{array}{l}34068 \\
124\end{array}$ & 13956 & $\begin{array}{l}752 \\
0.93\end{array}$ & 25732 & 34068 \\
\hline Cost per woman treated & 22.11 & 34.26 & 40.32 & 20.05 & $\begin{array}{l}0.93 \\
22.11\end{array}$ & $\begin{array}{l}1.96 \\
34.26\end{array}$ & $\begin{array}{l}1.24 \\
40.32\end{array}$ \\
\hline Cost per person treated & 11.93 & 21.82 & 26.27 & 14.54 & 11.93 & 21.82 & 26.27 \\
\hline Cost per congenital syphilis case averted & 158 & 86 & 177 & & & & \\
\hline Cost per adverse outcome averted & 44 & 62 & 89 & 187 & 181 & 280 & 300 \\
\hline Cost per DALY saved & 3.97 & 9.84 & 9.57 & 10.56 & 11.28 & 17.48 & 18.73 \\
\hline
\end{tabular}

context of high prevalence of HIV and syphilis, economies of scope can be achieved, rendering both PMTCT and syphilis prevention activities more cost effective. Economies of scope arise when an increase in the range of services provided leads to lower average costs than when the services are provided independently of each other. Although the PMTCT has an important role in antenatal care in high HIV prevalence areas, it is crucial that it does not divert resources away from cost effective, but less high profile, interventions. This study shows that on-site syphilis screening and treatment are extremely good value for money and, given the devastating impact of untreated maternal syphilis in pregnancy, are urgently in need of scaling up to national level in subSaharan African countries.

\section{ACKNOWLEDGEMENTS}

We are grateful to the principal secretary, Ministry of Health, the manager of the National AIDS Control Programme, and the director general of the National Institute for Medical Research (NIMR), Tanzania, for permission to conduct and publish the results of this study. We thank the following staff members of Makongoro Clinic for their assistance with the costing data: Neema Fimbo, Justa Lucas, Leah Bukongoro, Christina Simon, Mr Kihayi. We gratefully acknowledge the assistance of the labour ward teams at Bugando Medical Centre, Sekou Toure Regional Hospital, Sengerema Designated District Hospital, and the statistical and laboratory staff of NIMR, Mwanza. We are grateful to David Ross for his advice, to Isabelle Sinha for assisting with data entry, and to Tamara Hurst for her assistance with the financial records. The valuable comments contributed by the reviewers were highly appreciated.

This study was funded by the Wellcome Trust through a training fellowship in clinical tropical medicine (DWJ). Richard Hayes and Helen Weiss receive support from the Medical Research Council. The UK Department for International Development (DFID) supports policies, programmes and projects to promote international development. DFID provided funds for this study as part of that objective but

\section{Key messages}

- This study contributes to the existing literature on the cost effectiveness of antenatal syphilis screening and treatment by using empirically measured data on the benefits to the infants (adverse outcomes of pregnancy averted), rather than modelled estimates using historical incidence data. It is the first paper to use empirical data and a generic outcome indicator (DALY) to compare the cost effectiveness of antenatal syphilis screening with other maternal-child health interventions.

- Antenatal syphilis screening and treatment are shown to be very cost effective. The economic cost is $\$ 1.44$ per women screened and the cost effectiveness is $\$ 10.56$ per DALY saved. This compares favourably with other child health interventions and higher profile activities such as the prevention of mother to child transmission of HIV (PMTCT).

- On-site syphilis screening and treatment are extremely good value for money, and, in situations of high HIV and syphilis prevalence, important synergies between antenatal syphilis screening and HIV prevention can be exploited. Antenatal syphilis screening and treatment provides an opportunity to identify and counsel couples who may be at increased risk for HIV infection. Also, where syphilis screening, or PMTCT, is already in place, it may be possible to provide PMTCT and antenatal syphilis screening in combination at a lower cost than providing the two independently. 
the views and opinions expressed are those of the authors alone. FTP and LK are members of the HIVTools Research Group, LSHTM. The authors are members of the following DFID/LSHTM Knowledge Programmes: Tuberculosis programme; HIV/AIDS and Sexually Transmitted Infections; Health Economics and Financing Programme.

\section{CONTRIBUTORS}

FT-P collected cost data and undertook the cost and cost effectiveness analysis, and took the lead in writing the paper; DW-J, principal investigator for the two Mwanza studies of syphilis in pregnancy producing effectiveness results, study design, and data analysis, collected cost data, participated in writing the paper; KM assisted with the intervention data collection and supervised the ANC team in Mwanza; LK, study design, supervision of collection and analysis of the cost and cost effectiveness study, participated in writing the paper; LN participated in the cost data collection and conducted field work for the Mwanza studies; HW worked on the data analysis and participated in writing the paper; JC assisted in the study design and supervised the laboratory work in Tanzania; JT supervised the data management and analysis in Mwanza; FL started the intervention, trained staff, and participated in the cost data collection; BG assisted in data collection and supervised delivery field teams; DM assisted with the study design and participated in writing the paper; $\mathrm{RH}$ assisted in the study design, analysis, participated in writing the paper.

\section{Authors' affiliations}

F Terris-Prestholt, D Watson-Jones, L Kumaranayake, H Weiss, J Todd,

D Mabey, R Hayes, London School of Hygiene and Tropical Medicine, London, UK

D Watson-Jones, J Changalucha, J Todd, National Institute for Medical Research, Mwanza, Tanzania

K Mugeye, L Ndeki, Municipal Office of Health, Mwanza, Tanzania

F Lisekie, African Medical and Research Foundation, Mwanza,

Tanzania

B Gumodoka, Bugando Medical Centre, Mwanza, Tanzania

\section{REFERENCES}

1 Dabis F, Newell M, Fransen L, et al. Prevention of mother-to-child transmission of HIV in developing countries: recommendations for practice. Health Policy Planning 2000;15:34-42.

2 Marseille E, Kahn JG, Mmiro F, et al. Cost effectiveness of single-dose nevirapine regimen for mothers and babies to decrease vertical HIV-1 transmission in sub-Saharan Africa. Lancet 1999:354:803-9.

3 World Bank. World Development Report 1993: investing in health. New York: World Bank, 1993.

4 Gloyd S, Chai S, Mercer MA. Antenatal syphilis in sub-Saharan Africa: missed opportunities for mortality reduction. Health Policy Planning $2001 ; 16: 29-34$.

5 World Health Organization. Global prevalence and incidence of selected curable sexually transmitted infections: syphilis. Geneva: WHO, Geneva, 2001.
6 Radolf J, Sanchez P, Schultz K, et al. Congenital syphilis. In: Holmes K, Sparling P, Mardh P, eds. Sexually transmitted diseases, 3rd ed. New York: McGraw-Hill, 1998

7 Finelli L, Berman S, Koumans E, et al. Bacterial diseases: congenital syphilis. Bull World Health Organ 1998;76(Suppl 2):S126-8.

8 Watson-Jones D, Gumodoka B, Weiss H, et al. Syphilis in pregnancy in Tanzania II. The effectiveness of antenatal syphilis screening and single dose benzathine penicillin treatment for the prevention of adverse pregnancy outcomes. J Infect Dis 2002;186:948-57.

9 Hira SK, Bhat GJ, Chikamata DM, et al. Syphilis intervention in pregnancy: Zambian demonstration project. Genitourin Med 1990;66:159-64.

10 Jenniskens F, Obwaka E, Kirisuah S. Syphilis control in pregnancy: decentralization of screening facilities to primary care level, a demonstration project in Nairobi, Kenya. Int J Gynaecol Obstet 1995;48(Suppl):S121-8.

11 Fonck K, Claeys P, Bashir F, et al. Syphilis control during pregnancy: effectiveness and sustainability of a decentralized program. Am J Public Health 2001;91:705-7.

12 Temmerman M, Mohamedali F, Fransen L. Syphilis prevention in pregnancy: an opportunity to improve reproductive and child health in Kenya. Health Policy Planning 1993;8:122-7.

13 Mayaud P, Uledi E, Cornelissen L, et al. Risk scores to detect cervical infections in urban antenatal clinic attenders in Mwanza, Tanzania. Sex Transm Infect 1998;74(Suppl 1):S139-45.

14 Kumaranayake L, Pepperall J, Goodman H, et al. Costing guidelines for HIV prevention strategies, I. Geneva: UNAIDS, 2000.

15 Watson-Jones D, Changalucha J, Gumodoka B, et al. Syphilis in pregnancy in Tanzania I. The impact of maternal syphilis on outcome of pregnancy. J Infect Dis 2002; 186:940-7.

16 McDermott J, Steketee R, Larsen S, et al. Syphilis-associated perinatal and infant mortality in rural Malawi. Bull World Health Organ 1993:71:773-780.

17 Watson-Jones D. Impact of syphilis on outcome of pregnancy and evaluation of syphilis screening strategies for the reduction of adverse pregnancy outcomes in Mwanza, Tanzania, PhD Thesis. London: London School of Hygiene and Tropical Medicine, University of London, 2001.

18 Fox-Rushby J, Hanson K. Calculating and presenting disability adjusted life years (DALYs) in cost effectiveness analysis. Health Policy Planning $2001 ; 16: 326-31$

19 Murray CJ, Lopez A. Global mortality, disability, and the contribution of risk factors: Global burden of disease study. Lancet 1997;349:1436-42.

20 AbouZahr C, Vaughan A. Assessing the burden of sexual and reproductive illhealth: questions regarding the use of disability-adjusted life years. Bull World Health Organ 2000;78:655-66.

21 Mclntire D, Bloom S, Casey B, et al. Birth weight in relation to morbidity and mortality among newborn infants. N Engl J Med 1999:340:1234-8.

22 McCormick $M$. The contribution of low birth weight to infant mortality and childhood morbidity. N Engl J Med 1983;31:82-90.

23 Greenwood A, Armstrong J, Byass P, et al. Malaria chemoprophylaxis, birth weight and child survival. Trans R Soc Trop Med Hyg 1992;86:483-5.

24 Yasmin S, Osrin D, Paul E, et al. Neonatal mortality of low-birth-weight infants in Bangladesh. Bull World Health Organ $2001 ; 79: 608-14$.

25 Chan M. Low birth-weight. In: Stanfield P, Brueton M, Chan MS, et al, eds. Diseases of Children in the Subtropics and Tropics, 4th ed. London: Edward Arnold, 1991.

26 Gilson L, Mkanje R, Grosskurth H, et al. Cost-effectiveness of improved treatment services for sexually transmitted diseases in preventing HIV-1 infection in Mwanza Region, Tanzania. Lancet 1997;350:1805-9.

27 Varley RC, Tarvid J, Chao D. A reassessment of the cost-effectiveness of water and sanitation interventions in programmes for controlling childhood diarrhoea. Bull World Health Organ 1998;76:617-31.

28 Walker N, Schwartlander B, Bryce J. Meeting international goals in child survival and HIV/AIDS. Lancet 2002;360:284-9. 